\title{
Malignant peripheral nerve sheath tumours and neurofibromatosis 1: A case series and recommendations for care
}

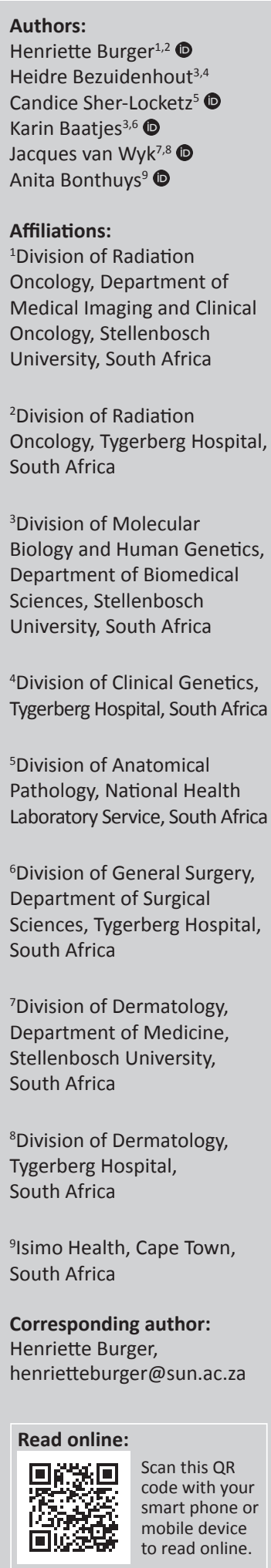

Background: The incidence of malignant peripheral nerve sheath tumours (MPNST) in patients with neurofibromatosis 1 (NF1) is significantly higher than that of the general population. NF1-associated MPNST occur at a younger age and carry a worse prognosis than sporadic MPNST.

Aim: This case series describes four cases of MPNST in patients with NF1.

Setting: The study was performed in a public academic hospital in the Western Cape province of South Africa.

Method: Demographics, disease status, histopathology, treatment and outcome data were collected retrospectively from medical charts and through review of histological slides.

Results: The median age was 36.5 years. All tumours were $>5 \mathrm{~cm}$ at presentation and located on the trunk. One patient presented with metastatic disease. There was a mean delay of 3.5 months from presentation to initiation of treatment. Three patients underwent wide excision, with one receiving adjuvant chemotherapy and radiotherapy. At a median follow-up of 20 months from histological diagnosis only one patient was alive in clinical remission. Two patients had succumbed to progressive disease at 8 and 16 months from diagnosis and one patient with terminal metastatic disease was lost to follow-up.

Conclusion: In this series the patients presented with advanced, often unresectable lesions for which single modality therapy was not curative. An adult NF1 health surveillance guideline for resource-constrained environments could lead to early diagnosis and treatment of MPNST and other complications in NF1 patients.

\section{Introduction}

Neurofibromatosis 1 (NF1) is a relatively common, autosomal dominant, neuro-cutaneous genetic disorder caused by a germline mutation in the NF1 tumour suppressor gene on chromosome 17q11. ${ }^{1}$ The disorder is associated with the development of several benign and malignant neoplasms, of which malignant peripheral nerve sheath tumours (MPNST) are the most common malignancy. ${ }^{2}$ The incidence of MPNST in patients with NF1 is more than 4000 times that of the general population. ${ }^{3}$ MPNST associated with NF1 occur at a younger age, are multicentric and carry a worse prognosis than sporadic MPNST., Importantly, it is the leading cause of early death in patients with NF1. ${ }^{5}$ Clinical features of NF1 usually develop in childhood, but MPNST is most prevalent between the age of 20 and 40 years. ${ }^{4}$ Currently no adult NF1 surveillance programme exists in the Western Cape public health system that could lead to the timely diagnosis and management of these tumours. This case series describes the clinico-pathologic characteristics, treatment and outcomes of four cases of MPNST in patients with NF1 referred to the sarcoma multidisciplinary team (SMDT) at Tygerberg Academic Hospital (TBAH), between 2013 and 2016.

\section{Methods}

The medical records of four patients diagnosed with NF1-associated MPNST were retrospectively reviewed by the first author. Data pertaining to demographics, tumour stage, histology, pertinent

Dates: Received: 05 June 2018 | Accepted: 20 June 2018 | Published: 20 Aug. 2018

How to cite this article: Burger $\mathrm{H}$, Bezuidenhout $\mathrm{H}$, Sher-Locketz $\mathrm{C}$, Baatjes K, Van Wyk J, Bonthuys A. Malignant peripheral nerve sheath tumours and neurofibromatosis 1: A case series and recommendations for care. S. Afr. j. oncol. 2018;2(0), a46. https://doi.org/10.4102/ sajo.v2i0.46

Copyright: @ 2018 . The Authors. Licensee: AOSIS. This work is licensed under the Creative Commons Attribution License. 
TABLE 1: Clinical, histological, treatment and outcome data.

\begin{tabular}{|c|c|c|c|c|}
\hline Variable & Patient 1 & Patient 2 & Patient 3 & Patient 4 \\
\hline Gender & Male & Male & Female & Female \\
\hline Age & 36 & 37 & 38 & 33 \\
\hline Family history of NF1 & No & Yes & No & No \\
\hline Timing of NF1 diagnosis & Pre-MPNST & Pre-MPNST & Pre-MPNST & Post-MPNST \\
\hline Speciality presented to & Dermatology & Neurosurgery/orthopaedic surgery & General surgery & Urology \\
\hline Primary site & Chest wall & Brachial plexus/supraclavicular fossa & Chest wall & Retroperitoneum \\
\hline Scoliosis & Yes & No & No & Yes \\
\hline AJCC stage group (2010) & 3 (T2aN1M0G3) & 3 (T2bNOMOG3) & 2B (T2bNOMOG2) & 4 (T2bNOM1G3) \\
\hline Surgery & WLE & Excision & WLE & Unresectable \\
\hline Heterologous differentiation & Smooth muscle & Smooth muscle, angiosarcomatous & Skeletal muscle & Smooth muscle \\
\hline FNCLCC grade & 3 & 3 & 2 & 3 \\
\hline Excision margins & Negative & Positive & Negative & N/A \\
\hline Radiotherapy & No & $\begin{array}{l}\text { Palliative CRT } 30 \text { Gray in } 10 \text { fractions, } \\
\text { weekly Adriamycin } \\
\text { Palliative RT } 8 \text { Gray in } 1 \text { fraction }\end{array}$ & $\begin{array}{l}\text { Adjuvant EBRT } \\
60 \text { Gray in } 30 \text { fractions }\end{array}$ & No \\
\hline Chemotherapy & No & Palliative§ & Adjuvant & Palliative§ \\
\hline Time to treatment $\dagger$ & $2 m$ & Simultaneous & $4 m$ & $2 \mathrm{~m}$ \\
\hline Time to PD & $3 \mathrm{~m}$ & $2 \mathrm{~m}$ & $\mathrm{~N} / \mathrm{A}$ & $2 \mathrm{~m}$ \\
\hline OS & $24 \mathrm{~m}$ (lost to FU) & $16 \mathrm{~m}$ & $26 \mathrm{~m}$ (alive with CR) & $8 \mathrm{~m}$ \\
\hline
\end{tabular}

NF1, neurofibromatosis 1; MPNST, malignant peripheral nerve sheath tumour; $m$, months; WLE, wide local excision; PD, progressive disease; SD, stable disease; OS, overall survival; AJCC, American Joint Committee on Cancer; FNCLCC, Fédération Nationale des Centres de Lutte Contre le Cancer; RT, radiotherapy; FU, follow-up; EBRT, external beam radiotherapy; CRT, chemoradiotherapy; TRV, transverse; CC, cranio-caudal; AP, anterior-posterior; $C R$, complete remission; N/A, not applicable.

$\dagger$, From histological diagnosis; $\$$, from first treatment; $\S$, adriamycin q3 weekly $\times 6 ; \boldsymbol{\Phi}$, adriamycin and ifosfamide q4 weekly $\times 4$.

clinical features, treatment modalities, recurrence and survival were captured. In addition, the histological slides of each patient were reviewed.

\section{Ethical considerations}

Ethics approval of conformance with the Declaration of Helsinki was obtained from the University of Stellenbosch Health Research Ethics Committee, reference number C16/06/008.

\section{Cases}

Clinical, histological, treatment and outcome data are presented in Table 1.

\section{Patient 1}

A 36-year-old male, known with NF1, presented with an 8-month history of a rapidly enlarging soft tissue mass anterior to the sternum. Prior surgical history included excision of a large $(13.7 \mathrm{~kg})$ truncal neurofibroma. Examination revealed multiple superficial neurofibromas and café-au-lait (CAL) macules of his trunk, upper limbs and face. The sternal mass measured $10 \mathrm{~cm} \times 9 \mathrm{~cm}$, was fixed to the chest wall and was ulcerated. A mobile left sub-centimetre axillary node was palpated. A punch biopsy of the mass showed features of a pleomorphic sarcoma. Fine needle aspiration biopsies of the left axillary node proved to be insufficient for analysis. Staging computer tomography (CT) showed a large, fungating sternal skin tumour $(5.8 \mathrm{~cm} \times 7.9 \mathrm{~cm})$ with irregular enhancement and central necrosis (Figure 1). It was indistinguishable from the medial aspect of the right pectoralis muscle, but no bone erosion was noted. Multiple bilateral sub-centimetre axillary nodes were present as well as one node in the right axilla measuring $1.6 \mathrm{~cm}$ in the short

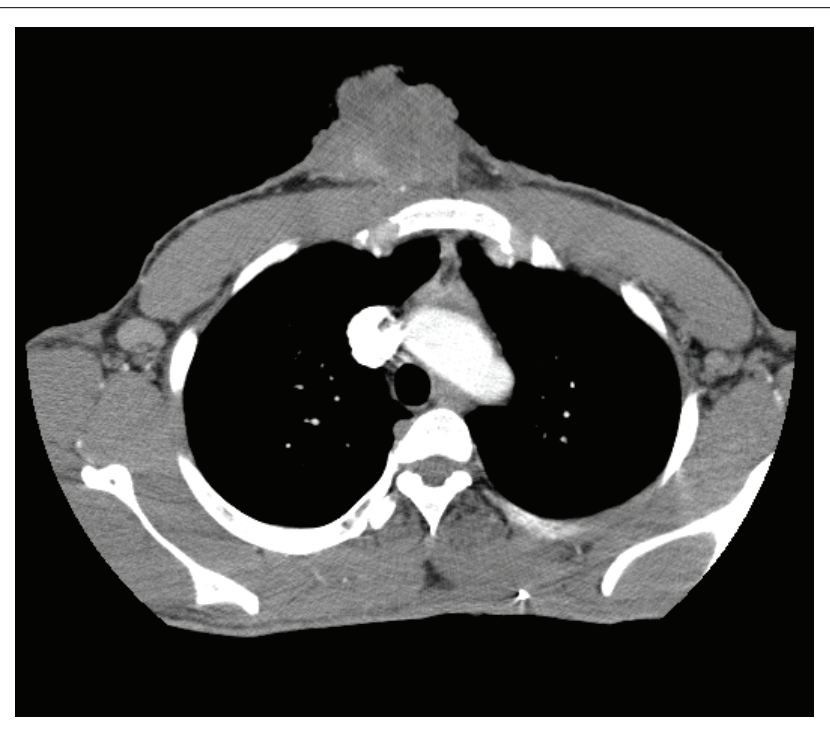

FIGURE 1: Patient 1 axial contrasted computed tomography through primary sternal malignant peripheral nerve sheath tumour showing pathological right axillary node.

axis. There were no visceral or bony metastases. Kyphosis of T6-8 with vertebral fusion was also noted. He was assessed by the SMDT and staged as a Stage III (T2aN1M0Gx) highgrade (HG) soft tissue sarcoma (STS). He underwent a wide local excision (WLE), which confirmed a $6.5 \mathrm{~cm} \times 6 \mathrm{~cm} \times 5 \mathrm{~cm}$ Grade 3 subcutaneous MPNST. The closest tumour-free margin measured $1.5 \mathrm{~mm}$ at depth. No axillary nodes were sampled. At post-operative SMDT review the node in the right axilla had enlarged, now measuring $2 \mathrm{~cm} \times 2 \mathrm{~cm}$. Fine needle aspiration biopsies revealed only reactive lymphocytes. Adjuvant external beam radiotherapy (EBRT) to the tumour bed was offered to decrease the risk of local recurrence. However, the patient did not return for EBRT and only presented again 2 months later with further enlargement 
of the right axillary node, now measuring $4 \times 4 \mathrm{~cm}$. He was referred for an axillary nodal dissection but did not attend the appointment. The patient was recalled, and a right axillary nodal dissection was performed 5 months after his WLE. Histological examination confirmed a metastatic MPNST with extra-capsular extension. The patient was offered adjuvant EBRT to the primary tumour bed followed by adjuvant chemotherapy but defaulted further treatment. He re-presented 16 months after his axillary dissection with a 3-month history of bilateral painful axillary masses and severe shortness of breath. Investigations revealed progressive disease with ulcerated axillary nodal recurrences, malignant pleural and pericardial effusions, and pleural metastases causing superior vena cava compression. His symptoms were palliated with a pleural tap and mediastinal and axillary EBRT. The patient was lost to follow-up 24 months from diagnosis and is presumed to have passed away.

\section{Patient 2}

A 37-year-old male, known with NF1, presented with a 3-year history of a $10 \mathrm{~cm} \times 10 \mathrm{~cm}$ enlarging mass of the right shoulder with paraesthesia and motor weakness of the arm. He was diagnosed with NF1 at the age of 15 and was referred to TBAH for genetic counselling. Both his father and sister had NF1 and his 3-year-old son had CAL macules. Magnetic resonance imaging (MRI) of the chest showed an $11 \mathrm{~cm} \times 8 \mathrm{~cm}$ heterogeneously enhancing mass in the right supraclavicular area suggestive of a MPNST arising from the brachial plexus and eroding the first rib (Figure 2). Deep neurofibromas were noted in the spinal cord at the levels of C6 and C7. The mass was resected in fragments that showed a HG MPNST arising from a plexiform neurofibroma. All resection margins were positive. The pathological stage was Stage III (pT2bN0M0G3). Post-operatively the patient had complete right-sided brachial



FIGURE 2: Patient 2 gadolinium-enhanced magnetic resonance imaging of primary tumour. plexus fallout. The patient was not referred to the SMDT for consideration of possible adjuvant therapy at this point. The patient returned 2 months post-operatively with painful recurrence $(14 \mathrm{~cm} \times 8 \mathrm{~cm})$ of the supraclavicular mass as well as dyspnoea. Imaging showed a recurrence of the right supraclavicular lesion extending intrapleurally, with a right pleural effusion and extradural extension into the spinal canal but without spinal cord compromise. No visceral or mediastinal metastases were noted. The recurrence was deemed unresectable and the patient was referred to the SMDT for management planning. The patient received palliative EBRT with weekly chemotherapy. Chest CT 2 months later showed reduction in the size of the primary tumour but revealed new lung metastases. The patient was given palliative chemotherapy. Restaging chest CT showed progression of the lung metastases but regression of the brachial plexus mass. At 15 months from diagnosis the patient developed significant local airway compression because of enlargement of the supraclavicular mass. A single fraction of palliative EBRT was administered and the patient demised 16 months from MPNST diagnosis.

\section{Patient 3}

A 38-year-old female, known with NF1, was referred with an 8 $\mathrm{cm} \times 8 \mathrm{~cm}$ mass of the right costal margin, which had been slowly enlarging since her previous pregnancy 2 years before. She reported noting superficial neurofibromas and CAL macules on her trunk, face and limbs since the age of 15 but was only formally diagnosed with NF1 at the age of 35 (Figure 3). She reported marked increase in the number of lesions during her previous two pregnancies. A core biopsy of the chest wall mass showed MPNST. Staging MRI of the chest revealed a 4.2 $\mathrm{cm} \times 5.6 \mathrm{~cm} \times 4.7 \mathrm{~cm}$ heterogeneous mass lateral to the 9 th and 10 th ribs. The mass was noted to protrude into the intercostal space but did not cause bony erosion or peritoneal invasion. She underwent a WLE with the closest tumour-free margin being reported as $1 \mathrm{~mm}$ at depth. Chest CT showed no lung metastases. At this point she was discussed at the SMDT and



FIGURE 3: Patient 3 skin manifestations of neurofibromatosis 1. 
staged as Stage IIB (T2bN0M0G2). She received adjuvant EBRT to the tumour bed and adjuvant chemotherapy without significant complications. Clinical and radiological review at 26 months from diagnosis showed complete remission.

\section{Patient 4}

A 33-year-old female presented 2 months post-partum with a large left-flank mass that became evident during the final month of her pregnancy. She was known with hypertension but was not aware of having NF1. She complained of abdominal pain, loss of appetite, post-prandial vomiting, dysuria and significant weight loss. On examination she was European Collaborative Oncology Group Performance Status 2 and in a wheelchair. She had a palpable, painful left-flank mass crossing the midline and cutaneous stigmata of NF1, including CAL macules and superficial neurofibromas over her trunk, limbs and face. These skin lesions had been present since childhood and had increased in number and size after menarche and during her pregnancies but were asymptomatic. AbdominalCT showed a $15 \mathrm{~cm} \times 16 \mathrm{~cm} \times 15 \mathrm{~cm}$ retroperitoneal mass effacing the psoas muscle and displacing the left kidney. The mass extended to the left iliac wing and the abdominal wall muscles. No hydronephrosis or visceral, bony or nodal metastases were seen. A transcutaneous core biopsy of the mass showed a spindle cell tumour, not otherwise specified, and a repeat biopsy was recommended. It should be noted that the pathologist was not made aware of her NF1 diagnosis. Chest CT showed a right apical soft tissue mass in keeping with a lung metastasis as well as a small left pleural effusion. Repeat abdominal CT showed disease progression with an increase in the size of the tumour as well as new onset left hydronephrosis and extensive tumour deposits in the peritoneum, 12th rib and left iliac muscle. An open biopsy was performed and showed a HG STS most likely in keeping with an MPNST. She was staged as Stage IV (T2bN0M1G3) HG MPNST with lung metastases. She received palliative chemotherapy. Restaging CT abdomen after four cycles showed no change in the size of the tumour but increased internal necrosis as well as a new left pleural effusion. Despite this the chemotherapy provided significant symptom relief and improvement in performance status and she completed six cycles. She succumbed to progressive disease 11 weeks later at 8 months from diagnosis.

\section{Discussion}

\section{Neurofibromatosis 1}

Estimated birth incidences for NF1 of 1 in 2500 to 1 in 3000 have been reported. ${ }^{6,7}$ Approximately $50 \%$ of cases are familial, being inherited in an autosomal dominant pattern, and in $50 \%$ the mutation arises de novo. The condition is fully penetrant, with all individuals carrying a mutation expressing the disorder. Clinical manifestations can however be variable even within families and develop with age. ${ }^{8}$ In our series only one of the patients reported a parent or sibling with NF1. This may not be a true reflection of the patterns of inheritance as it is quite common for patients attending TBAH to be unaware of their familial medical history. Of the 16 children born between the four patients, six have subsequently been assessed by the division of clinical genetics and only one has been diagnosed with NF1.

The clinical manifestations of NF1 most commonly affect the skin, skeletal and nervous systems. Table 2 details these

TABLE 2: Clinical manifestations of neurofibromatosis 1.,

\begin{tabular}{|c|c|c|}
\hline Organ system & Clinical manifestations & Complications \\
\hline \multirow[t]{2}{*}{ Skin } & Café-au-lait macules & Disfigurement \\
\hline & Skin-fold freckling & \\
\hline \multirow[t]{2}{*}{ Peripheral nerves } & Cutaneous neurofibromas & $\begin{array}{l}\text { Disfigurement, tenderness, pruritus, traumatic injury, low potential for malignant } \\
\text { transformation }\end{array}$ \\
\hline & Plexiform neurofibromas & $\begin{array}{l}\text { Soft tissue infiltration, bony hypertrophy, spinal cord or nerve root compression, } \\
\text { potential for malignant transformation }\end{array}$ \\
\hline \multirow[t]{4}{*}{ Central nervous system } & Cerebral and optic pathway gliomas & Raised ICP, visual impairment, squint \\
\hline & Cerebrovasculopathy & Cerebrovascular events \\
\hline & Cognitive impairment & Learning difficulty, behavioural problems, impaired insight into medical care \\
\hline & Psychological distress & Depression, anxiety \\
\hline \multirow[t]{3}{*}{ Skeletal } & Sphenoid wing dysplasia & Skull deformity, disfigurement, exophthalmos \\
\hline & Scoliosis & $\begin{array}{l}\text { Nerve root and spinal cord compression, respiratory compromise, muscle spasm, } \\
\text { disfigurement }\end{array}$ \\
\hline & Bowing of long bones ( \pm congenital tibial pseudo arthrosis) & Fracture, disfigurement, may require amputation \\
\hline \multirow[t]{4}{*}{ Cardiovascular } & $\begin{array}{l}\text { Vasculopathy (renal artery stenosis, coarctation of the } \\
\text { aorta, pulmonary artery stenosis) }\end{array}$ & Hypertension, cardiac failure \\
\hline & Essential hypertension & End organ damage \\
\hline & Congenital heart defects & Cardiac failure, arrhythmias \\
\hline & Peripheral glomus tumours & Pain \\
\hline Gastrointestinal & GIST & Obstruction, bleeding \\
\hline Endocrine & Pheochromocytoma & Hypertension \\
\hline Growth & Short stature, macrocephaly & Disfigurement \\
\hline Haematological & Leukaemia & Significant multi-system morbidity and mortality \\
\hline
\end{tabular}

Source: Ferner et al. and Korf

ICP, intra-cranial pressure; GIST, gastrointestinal stromal tumour. 
BOX 1: Diagnostic criteria for neurofibromatosis $1 .{ }^{10}$

Presence of two or more of the following features:

- Six or more café-au-lait macules over $5 \mathrm{~mm}$ in greatest diameter in prepubertal individuals and over $15 \mathrm{~mm}$ in greatest diameter in post-pubertal individuals

- Two or more neurofibromas of any type or one plexiform neurofibroma

- Freckling in the axillary or inguinal regions

- Optic glioma

- Two or more Lisch nodules (iris hamartomas)

- A distinctive osseous lesion such as sphenoid dysplasia, thinning of long bone cortex causing anterolateral tibial bowing with or without pseudarthrosis

- A first-degree relative (parent, sibling or offspring) with neurofibromatosis 1 as defined by the above criteria

Source: National Institutes of Health

manifestations according to organ system along with possible related complications. ${ }^{7,9}$ The clinical diagnosis of NF1 is based on the diagnostic criteria developed by the National Institutes of Health Consensus Development Conference and published in 1988 (Box 1). ${ }^{10}$ Two or more diagnostic criteria need to be met to make a conclusive clinical diagnosis and can be used from late childhood. Nearly all patients develop characteristic clinical features before the age of 8 years. ${ }^{4}$

In the South African setting the diagnosis is usually established based on clinical manifestations, with genetic testing being reserved for clinically uncertain cases and antenatal diagnoses. This can be ascribed to the high cost of genetic testing resulting from the large gene size and large number of different known mutations and to the fact that a clinical diagnosis based on non-invasive evaluation usually can be made before serious complications occur. In our series, three patients were aware of their NF1 diagnosis, but only one had received formal genetic counselling and advice about possible future medical complications related to the condition. Importantly, none of the four patients were part of a follow-up programme.

Pregnancy in the NF1 patient may require specialist supervision because of higher rates of pregnancy-associated hypertension and perinatal complications including preterm labour, increased caesarean section rates and intrauterine growth restriction. ${ }^{11}$ In addition, hormonal changes because of pregnancy have been reported to be associated with an increase in the number and size of neurofibromas as was seen in Patients 3 and 4. This could lead to complications like spinal cord compression and pelvic outlet obstruction..$^{12,13}$ All pregnant NF1 patients should receive genetic counselling as the risk of having a child with NF1 is $50 \%$ with each pregnancy and clinical manifestations can be widely variable. ${ }^{13}$

\section{Malignant peripheral nerve sheath tumour}

In the general population, MPNST is a rare disorder with a reported incidence of $0.001 \%$. Patients with NF1 however have an estimated lifetime risk of being diagnosed with MPNST of $8 \%-13 \%$. Between $20 \%$ and $50 \%$ of MPNST are NF1-associated. ${ }^{14}$ MPNST show no gender predilection and typically present at an earlier age when compared with other STSs, which tend to predominate in patients beyond the sixth decade. . $5,16,17,18$ MPNST may arise in a pre-existing neurofibroma or de novo in any peripheral nerve. They are however more likely to be associated with plexiform neurofibromas, particularly those associated with the brachial or sacral plexuses. ${ }^{19}$ NF1-associated lesions tend to be located on the trunk rather than the extremities as with non-NF1 MPNST. ${ }^{17}$ Lesions typically present as palpable, painless, enlarging soft tissue masses often larger than $5 \mathrm{~cm} \cdot{ }^{15,20}$ Patients may also present with symptoms specific to the involved nerve, including pain, paraesthesia and motor deficits. ${ }^{21}$ The onset of new, progressive or persistent pain in a pre-existing neurofibroma should be thoroughly investigated. In this series patients were referred from secondary-level healthcare facilities with complaints of enlarging masses, local neuropathic symptoms, ulceration and systemic symptoms. They were referred to the divisions of dermatology, neurosurgery, general surgery and urology, respectively, which is consistent with internationally reported trends of diverse referral patterns. Three patients had Stage III or IV disease and all had tumours larger than $5 \mathrm{~cm}$ that could conceivably have been diagnosed at an earlier stage. The possible relationship between hormonal changes during pregnancy and malignant transformation of benign neurofibromas in NF1 patients has been explored in a small number of case reports and preclinical studies, but a definitive association has not been proven. ${ }^{12,13}$

From a diagnostic point of view MPNST is a malignancy arising (1) from a peripheral nerve, (2) from a pre-existing benign peripheral nerve sheath tumour (usually neurofibroma) or (3) in patients with NF1 disease..$^{22}$ Outside these scenarios extensive immunohistochemical tests, ultra-structural studies and molecular testing is required to confidently distinguish MPNST from other STSs, because of the diverse morphology of these tumours. The diverse morphology can include heterologous differentiation, which is relatively more common in the setting of NF1. ${ }^{23,24,25}$ Diagnosis outside the setting of NF1 can therefore be challenging especially on limited tissue samples and it is therefore imperative that communication between the surgeon and pathologist is maintained regarding the history of NF1. This can avert the need for costly repeat biopsies and additional immuno-histochemical and molecular investigation. The most widely accepted grading system for STSs is the French FNCLCC (Fédération Nationale des Centres de Lutte Contre le Cancer) system, which is three-tiered and based on tumour differentiation, amount of necrosis and mitotic activity. The system has not been proven to be of prognostic relevance in MPNST, with most lesions being regarded as HG 'by default' and some authors suggesting a two-tiered grading system that takes NF1 status into account. ${ }^{16,24,26}$ The pathological findings of this series are included in Table 1.

\section{Management of malignant peripheral nerve sheath tumour}

The staging and management of MPNST follows the guidelines for adult-type STSs and relies heavily on the collaboration of a multidisciplinary team of specialists from the time of diagnosis to ensure disease control. ${ }^{27}$ Surgical resection with clear margins remains the mainstay of curative treatment, with the addition of radiotherapy (RT) and chemotherapy effecting a local control advantage but not a 
proven survival benefit. $28,29,30,31,32$ Neo-adjuvant and adjuvant RT have also allowed for higher rates of limb salvage..$^{30,33}$

Patient outcomes are summarised in Table 1. The three nonmetastatic patients all underwent WLE of their primary lesions by a general or orthopaedic surgeon. Despite TBAH having an established SMDT only one patient was discussed there prior to surgical intervention. All three had suboptimal $(<1 \mathrm{~cm})$ resection margins. One patient received adjuvant chemotherapy and RT. The remaining two had loco-regional recurrence at 2 and 3 months after surgery. Three patients received palliative chemotherapy, one upfront and two at time of progression. Two patients received palliative RT at time of progression. In this series there was a mean 3.5-month delay (range 2-6) from presentation to TBAH and initiation of treatment for MPNST. This constitutes unacceptable delays in the management of an aggressive malignancy.

MPNST is associated with higher rates of local recurrence, distant spread and worse tumour-related survival compared to other STS. ${ }^{17}$ In addition to advanced stage and recurrent lesions, size greater than $5 \mathrm{~cm}$, incomplete resection, $\mathrm{HG}$ and rhabdomyoblastic differentiation have been identified as poor prognostic factors. ${ }^{3,18,20,34}$ Overall survival (OS) rates of $45 \%$ - 55\% have been reported in modern series. ${ }^{17,34}$ The prognostic effect of NF1 on MPNST has been inconsistently reported, with some series reporting no effect on survival ${ }^{15,26,34,35}$ and others showing decreased survival outcomes in NF1 patients. ${ }^{3,16,17,20,36}$ A recent series reported 5 -year OS of $60 \%$ in sporadic MPNST versus 32\% in NF1associated disease. ${ }^{17}$ However, a meta-analysis of MPNST case series published between 1963 and 2012 including more than 1800 patients showed that prognosis was significantly worse in patients with NF1 but that the difference became less significant over the last decade of the study period (2001-2012). ${ }^{34}$ It is unclear whether the worse prognosis in NF1-associated MPNST was related to tumour biology or a result of clinical factors such as delayed presentation with increased risk of systemic dissemination and larger tumours with ensuing subtotal resection. The apparent improvement in recent outcomes could possibly be ascribed to earlier diagnosis because of NF1 surveillance programmes and the availability of treatment guidelines. ${ }^{20,34}$

\section{Recommendations for adult neurofibromatosis 1 surveillance}

Because NF1 is a complex disease with a wide variety of clinical manifestations, these patients present to different medical and surgical specialities. These may include general practitioners, paediatricians, neurologists, dermatologists, surgeons, obstetricians, geneticists and oncologists. ${ }^{37}$ Many individuals with NF1 lead healthy lives with only cosmetic concerns because of skin pigmentation and neurofibromas. Only a third of individuals with NF1 will ultimately have medical problems related to the disorder. Some of these problems will be mild and easily treatable and others will be more severe and need specialist or urgent attention. Therefore lifelong health surveillance is advised for specific, well-defined, potentially serious complications to aid early detection and timeous treatment. ${ }^{4}$

One of the challenges in the care of individuals with NF1 in South Africa is the absence of a structured surveillance service. Such a service would facilitate the diagnosis and registration of NF1 during childhood, continue to monitor affected individuals for complications and provide ongoing age-appropriate counselling about the condition, potential complications and the familial implications of the disease. Only one of the patients in the series had received prior genetic counselling as an adolescent, but this was not followed up. This lack of preventative management could be attributed to the mostly reactive role of the public healthcare system, lack of funds and the lack of communication and cooperation between the different levels and different disciplines in the public healthcare system. Without education and counselling, affected individuals often display delayed and inconsistent healthcare-seeking behaviour, which, in the case of an aggressive tumour like MPNST, can result in preventable morbidity and mortality.

The establishment and strengthening of structured surveillance programmes for NF1 patients in the paediatric setting is an essential foundation for adult NF1 care but falls outside the scope of this article. Those individuals who are treated for NF1-related complications during childhood are mostly retained in the system, but transition to adult care is often inconsistent. To our knowledge there is no formal surveillance protocol beyond late childhood for uncomplicated NF1 patients in the Western Cape public health system to facilitate lifelong healthcare management.

In Box 2 we propose a basic surveillance strategy for NF1 adults over the age of 18 years in resource-limited settings, considering current international guidelines and constraints in the public healthcare services. ${ }^{4,714,38}$ It outlines ongoing surveillance that includes regular focussed clinical examination by a primary healthcare clinician, patient education regarding potential complications of NF1 and information on symptoms that should prompt medical attention. It is proposed that the surveillance programme be patient-driven but that the necessary support be given in the form of information leaflets and contact details of NF1

BOX 2: Proposed surveillance guidelines for adult ( $>18$ years) neurofibromatosis 1 patients.

$$
\begin{aligned}
& \text { - Annual physical examination and symptom enquiry by a general physician } \\
& \text { - Skin examination for symptomatic neurofibromas } \\
& \text { - Neurological and spinal exam for symptoms and signs of deep plexiform } \\
& \text { - Blood promassure mand scoliosis } \\
& \text { - High index of suspicion and early referral of potential MPNST to tertiary referral } \\
& \text { service: Any new, progressive or persistent pain or rapid growth, either in } \\
& \text { pre-existing plexiform tumour or deep-seated locations, for example, branchial } \\
& \text { plexus or sciatic nerve } \\
& \text { - Advise to avoid radiotherapy as it may increase risk of MPNST } \\
& \text { - Patient education on inheritance patterns of NF1 and pregnancy-related } \\
& \text { - Psmplications } \\
& \text { - Monitoring of those who have abnormalities of the central nervous system, } \\
& \text { - Okeletal system or cardiovascular system by an appropriate specialist } \\
& \text { - Other studies (e.g. MRI) only as indicated on the basis of clinical signs or symptoms }
\end{aligned}
$$

Source: Adapted from Jett and Friedman, Ferner et al., Evans et al., Hersh

MPNST, malignant peripheral nerve sheath tumour; MRI, magnetic resonance imaging; NF1, neurofibromatosis 1 . 
specialist clinics to ensure effective and rapid diagnosis and management of complications should they arise. As a direct result of this collaboration, the TBAH Division of Medical Genetics has formulated a patient-centred information leaflet that explains the genetic cause and inheritance patterns, clinical manifestations and complications of NF1 as well as guidelines for surveillance of children and adults. ${ }^{39}$ The families of three of the patients have subsequently received counselling and screening for NF1.

There are currently no dedicated multidisciplinary NF1 specialist clinics in the public health sector beyond childhood. It is recommended that such clinics combine the expertise of neurologists, geneticists, ophthalmologists, endocrinologists, neurosurgeons, plastic surgeons, orthopaedic surgeons, soft tissue tumour surgeons, psychiatrists, dermatologists, radiologists, oncologists and pathologists. ${ }^{7,38}$ All this expertise co-exists in public tertiary health facilities and, although time and service constraints hinder them from having formal meetings, effective communication using technology and shared goals mean that 'virtual' NF1 clinics could be established without too much effort. Benefits of such a service would include (1) consensus diagnosis of difficult cases, (2) providing a contact point for expedited referral, diagnosis and management of complications of NF1, (3) multidisciplinary management of serious complications of NF1, (4) providing support and education for patients and their families and (5) overseeing public and professional education on NF1. The challenge lies in identifying champions to establish and lead such clinics in tertiary institutions. Until such collaborative efforts are adequately established, basic guidelines can be introduced in the form of a patient information leaflet to inform both patients and their primary healthcare workers about important surveillance activities that can reduce morbidity. A further recommendation is that all cases of suspected or confirmed MPNST in patients with NF1 be referred to an SMDT for staging and management before any definitive surgery is attempted. ${ }^{17,20}$

\section{Conclusions}

The prevalence of NF1 in South Africa is not well documented, but with a worldwide birth incidence as high as 1 in 3000 this condition likely affects a significant number of the population. Patients are usually diagnosed in childhood, but close followup does not continue into adulthood, when the risk of complications persists and the risk of MPNST in particular is at its highest. MPNST is a rare but aggressive malignancy with poor survival outcomes, particularly in NF1 patients. In this series the patients presented with advanced, often unresectable lesions for which single modality therapy was not curative. There is currently no surveillance programme for adult NF1 patients in the Western Cape public health system. The combination of centralised multidisciplinary adult NF1 clinics and a patient-driven health surveillance guideline as outlined here could lead to early diagnosis and treatment of MPNST and other complications in patients with NF1 in resource-constrained healthcare systems.

\section{Acknowledgements}

The authors would like to thank Professor H. Simonds, head of the Division of Radiation Oncology at Stellenbosch University and Tygerberg Hospital, for editorial revision.

\section{Competing interests}

The authors declare that they have no financial or personal relationships that may have inappropriately influenced them in writing this article.

\section{Authors' contributions}

$\mathrm{H}$. Burger was the project leader. H. Burger, H. Bezuidenhout, C.S-L., K.B., J.v.W. and A.B. contributed to the drafting and revision of the manuscript. All authors approved the final version.

\section{References}

1. Viskochil D, Buchberg $A, X u G$, et al. Deletions and a translocation interrupt a cloned gene at the neurofibromatosis type 1 locus. Cell. 1990;62:167-192. https://doi.org/10.1016/0092-8674(90)90252-A

2. Ferner R, Gutmann D. International consensus statement on malignant periphera nerve sheath tumours in neurofibromatosis 1. Cancer Res. 2002;62:1573-1577.

3. Ducatman B, Scheithauer B, Piepgras D, Reiman H, Ilstrup D. Malignant peripheral nerve sheath tumors. A clinicopathologic study of 120 cases. Cancer. 1986;57: 2006-2021. https://doi.org/10.1002/1097-0142(19860515)57:10<2006::AIDCNCR2820571022>3.0.CO;2-6

4. Jett K, Friedman J. Clinical and genetic aspects of neurofibromatosis 1 . Genet Med. 2010;12(1):1-11. https://doi.org/10.1097/GIM.0b013e3181bf15e3

5. Rasmussen $S$, Yang $Q$, Friedman J. Mortality in neurofibromatosis 1 : An analysis using U.S. death certificates. Am J Hum Genet. 2001;68(5):1110-1118. https:// doi.org/10.1086/320121

6. Evans D, Howard E, Giblin C, et al. Birth incidence and prevalence of tumour-prone syndromes: Estimates from a UK family genetic register service. Am J Med Genet A. 2010;152A(2):327-332. https://doi.org/10.1002/ajmg.a.33139

7. Ferner R, Huson $\mathrm{S}$, Thomas $\mathrm{N}$, et al. Guidelines for the diagnosis and management of individuals with Neurofibromatosis 1. J Med Genet. 2007;44:81-88. https:// doi.org/10.1136/jmg.2006.045906

8. Viskochil D. Genetics of neurofibromatosis 1 and the NF1 gene. J Child Neurol. 2002;17(18):562-572. https://doi.org/10.1177/088307380201700804

9. Korf B. Malignancy in neurofibromatosis type 1. Oncologist. 2000;5:477-485. https://doi.org/10.1634/theoncologist.5-6-477

10. National Institutes of Health Consensus Development Conference Statement: Neurofibromatosis. Arch Neurol 1988;45:575-578.

11. Terry A, Barker F, Leffert L, Bateman B, Souter I, Plotkin S. Neurofibromatosis type 1 and pregnancy complications: A population-based study. Am J Obstet Gynaecol. 2013;209(1):46e1-46e8.

12. Roth T, Petty E, Barald K. The role of steroid hormones in the NF1 phenotype: Focus on pregnancy. Am J Medi Genet Part A. 2008;146A:1624-1633. https://doi. org/10.1002/ajmg.a.32301

13. Chetty S, Shaffer B, Norton M. Management of pregnancy in women with genetic disorders: Part 2: Inborn errors of metabolism, cystic fibrosis, neurofibromatosis type 1 and Turner syndrome in pregnancy. Obstet Gynaecol Surv. 2011:66(12):765776. https://doi.org/10.1097/OGX.0b013e31823cdd7d

14. Evans D, Baser M, McGaughran J, Sharif S, Howard E, Moran A. Malignant peripheral nerve sheath tumours in neurofibromatosis 1. J Med Genet. 2002;39:311-314. https://doi.org/10.1136/jmg.39.5.311

15. Anghileri $M$, Miceli $R$, Fiore $M$, et al. Malignant peripheral nerve sheath tumours: Prognostic factors and survival in a series of patients treated at a single institution. Cancer. 2006;107(5):1065-1074.

16. Hagel C, Zils U, Peiper M, et al. Histopathology and clinical outcome of NF1 associated vs. sporadic malignant peripheral nerve sheath tumour. J Neurooncol. 2007;82(2):187. https://doi.org/10.1007/s11060-006-9266-2

17. Porter D, Prasad V, Foster L, Dall G, Birch R, Grimer J. Survival in malignant peripheral nerve sheath tumours: A comparison between sporadic and neurofibromatosis type 1-associated tumours. Sarcoma. 2009;2009:756395. https://doi.org/10.1155/2009/756395

18. Kim D, Murovic J, Tiel R, Moes G, Kline D. A series of 397 peripheral neural sheath tumours 30-year experience at Louisiana State University Health Sciences Centre. J Neurosurg. 2005;102:246-255. https://doi.org/10.3171/jns.2005.102.2.0246

19. Tucker T, Wolkenstein P, Revuz J, Zeller J, Friedman J. Association between benign and malignant peripheral nerve sheath tumours in NF1. 2005;65(2):205-11. 
20. Stucky C, Johnson K, Gray R, et al. Malignant peripheral nerve sheath tumours (MPNST): The Mayo Clinic Experience. Ann Surg Oncol. 2012;19:878-885. https:// doi.org/10.1245/s10434-011-1978-7

21. Ramanathan R, Thomas J. Malignant peripheral nerve sheath tumours associated with von Recklinghausen's neurofibromatosis. Eur J Surg Oncol. 1999:25:190-193. https://doi.org/10.1053/ejso.1998.0625

22. Le Guellec S, Decouvelaere AV, Filleron T, et al. Malignant peripheral nerve sheath tumour is a challenging diagnosis: A systematic pathology review, immunohistochemistry, and molecular analysis in 160 patients from the French Sarcoma Group Database. Am J Surg Pathol. 2016;40:896-908. https://doi. org/10.1097/PAS.0000000000000655

23. Fletcher C, Bridge J, Hogendoom P. WHO classification of tumours of soft tissue and bone. Lyon, France: IARC; 2013.

24. Rodriguez F, Folpe A, Giannini C, Perry A. Pathology of peripheral nerve sheath tumours: Diagnostic overview and update on selected problems. Acta Neuropathol. 2012;123:295-319. https://doi.org/10.1007/s00401-012-0954-z

25. Takeuchi A, Ushigome S. Diverse differentiation in malignant peripheral nerve sheath tumours associated with neurofibromatosis-1, an immunohistochemica and ultra-structural study. Histopathology. 2001;39:298-309. https://doi. org/10.1046/j.1365-2559.2001.01212.x

26. Zou C, Smith K, Liu J, et al. Clinical, pathological, and molecular variables predictive of malignant peripheral nerve sheath tumour outcome. Ann Surg. 2009;249(6):1014-1022. https://doi.org/10.1097/SLA.0b013e3181a77e9a

27. The ESMO/European Sarcoma Network Working Group. Soft tissue and visceral sarcomas: ESMO Clinical Practice Guidelines for diagnosis, treatment and follow-
up. Ann Oncol. 2014;25(Suppl 3):iii102-iii112. https://doi.org/10.1093/annonc/ mdu 254

28. Gachiani J, Kim D, Nelson A, Kline D. Surgical management of peripheral nerve sheath tumours. Neurosurg Focus. 2007;22(6):E13.

29. Levi A, Ross A, Cuartas E, Qadir R, Temple H. The surgical management of Symptomatic peripheral nerve sheath tumours. Neurosurgery. 2010;66(4):833840. https://doi.org/10.1227/01.NEU.0000367636.91555.70
30. Strander H, Tureson I, Cavallin-Stahl E. A systematic overview of radiation therapy effects in soft tissue sarcomas. Acta Oncol. 2003;42(5-6):516-531. https://doi. org/10.1080/02841860310014732

31. Pervaiz N, Colterjohn N, Farrokhyar F, Tozer R, Figuerdo A, Ghert M. A systematic meta-analysis of randomized controlled trials of adjuvant chemotherapy for localized resectable soft-tissue sarcoma. Cancer. 2008;113(3):573-581. https:// doi.org/10.1002/cncr.23592

32. Le Cesne A, Ouali M, Leahy $M$, et al. Doxorubicin-based adjuvant chemotherapy in soft tissue sarcoma pooled analysis of two STBSG-EORTC phase III clinical trials. Ann Oncol. 2014;25:2425-2432. https://doi.org/10.1093/annonc/mdu460

33. Davis M, O'Sullivan B, Turcotte R, et al. Late radiation morbidity following randomization to preoperative versus postoperative radiotherapy in extremity soft tissue sarcoma. Radiother Oncol. 2005;75:48-53. https://doi.org/10.1016/j. radonc.2004.12.020

34. Kolberg M, Høland M, Agesen T, et al. Survival meta-analyses for 1800 malignant peripheral nerve sheath tumour patients with and without neurofibromatosis Type-1. Neuro Oncol. 2013. https://doi.org/10.1093/neuonc/nos287

35. Hruban H, Shiu M, Senie R, Woodruff J. Malignant Peripheral nerve sheath tumours of the buttock and lower extremity: A study of 43 cases. Cancer. 1990;66: 1253-1265. https://doi.org/10.1002/1097-0142(19900915)66:6<1253::AlD1253-1265. https://doi.org/
CNCR2820660627>3.0.CO;2-R

36. Wong W, Hirose T, Scheithauer B, Schild S, Gunderson L. Malignant peripheral nerve sheath tumour: Analysis of treatment outcome. Int J Radiat Oncol Biol Phys. 1998;42(2):351-360. https://doi.org/10.1016/S0360-3016(98)00223-5

37. Hirbe A, Gutmann D. Neurofibromatosis type 1: A multidisciplinary approach to care. Lancet Neurol. 2014;13(8):834-843. https://doi.org/10.1016/S14744422(14)70063-8

38. Hersh J. Health supervision for children with neurofibromatosis. Paediatrics. 2008;121:633-642. https://doi.org/10.1542/peds.2007-3364

39. Division of Molecular Biology and Human Genetics, Stellenbosch University. Neurofibromatosis 1: Patient information sheet. 2016. 